\title{
PLACENTAL PARAMETERS AND FOETOMATERNAL HAEMORRHAGE
}

\author{
Sanghamithra V. Reddy', Muralidhar V. Pai ${ }^{2}$
}

1 Junior Resident, Department of Obstetrics and Gynaecology, Kasturba Medical College.

2 Professor and HOD, Department of Obstetrics and Gynaecology, Kasturba Medical College.

\begin{abstract}
BACKGROUND
ABSTRACT

Entry of foetal erythrocytes into maternal circulation before or during delivery is referred as Foetomaternal Haemorrhage (FMH). An Rh-D negative women when exposed to the cells of an Rh-D positive foetus, produces anti-D antibodies that causes complications like foetal anaemia, hydrops foetalis and neonatal jaundice. The Kleihauer-Betke test is a quantitative test, which uses the concept of differential resistance to acid by foetal and adult haemoglobin. As the potential risk factors can be determined by placental morphology and provide information on pregnancy outcome, they may provide information on incidence and amount of FMH also.

The objective of this study is to determine the relationship between placental parameters (weight and diameter) and the incidence as well as severity of Foetomaternal Haemorrhage (FMH). The secondary aim was to correlate FMH with maternal factors and neonatal anaemia.
\end{abstract}

\section{MATERIALS AND METHODS}

A total of 333 women who delivered in the hospital after 28 weeks of gestation were enrolled for the study. Maternal blood ( $2 \mathrm{~mL}$ ) was collected in EDTA bottle after the delivery of the baby. Samples were sent to Blood Bank. FMH was quantified by KleihauerBetke's test. The foetomaternal haemorrhage is calculated as: Number of foetal cells per high power field/Number of maternal cells per high power field x 2400.1 FMH $>2 \mathrm{~mL}$ was considered positive for FMH as per BSCH (British Committee for Standards in Haematology) guidelines. ${ }^{2}$ Placental parameters like weight (after trimming cord at $5 \mathrm{~mm}$ from the insertion point and clearing membranes, clots) and diameter were measured. A cut-off of $500 \mathrm{gms}$ for placental weight and $22 \mathrm{~cm}$ for placental diameter was taken. ${ }^{3,4,5}$ Actual birth weight and neonatal Haemoglobin ( $\left.\mathrm{Hb}\right)$ was noted.

BSCH = British Committee for Standards in Haematology.

\section{RESULTS}

Out of 333 subjects 48 (14.4\%) were positive for FMH (Group 1) and 285 (85.6\%) were negative for FMH (Group 2). The mean placental weight $(508.54 \pm 80.34 \mathrm{gm})$ and the mean placental diameter $(19.92 \pm 3.99 \mathrm{~cm})$ were significantly more in patients having positive FMH when compared to those with negative FMH. (P value $=0.0005$ for placental weight and $\mathrm{p}=0.005$ for placental diameter). When placental weight was $\geq 500$ gm more subjects were significantly positive for FMH (25.6\%) compared to when that was less than $500 \mathrm{gm}(7 \%)(\mathrm{P}$ value $=0.00)$. When the placental weight was between 600 and $699 \mathrm{gm}$, the odds of having FMH was 13.43 times more. When the placental diameter was $\geq 22 \mathrm{~cm}$, the incidence of positive FMH was significantly more (33.9\%) compared to when that was $<22 \mathrm{~cm}(10.2 \%)$ (P value $=0.00)$ and the odds of having positive FMH was 4.51 times. When the placental diameter was between 23 and $27 \mathrm{~cm}$, the odds of having FMH was 5 times more. When the FMH was $>2 \mathrm{~mL}$ both mean placental weight $(508.54 \pm 80.34 \mathrm{gm})$ and diameter $(19.92 \pm 4.00 \mathrm{~cm})$ were significantly higher when compared to lesser FMH. (P value 0.00 for both weight and diameter). Incidence of positive FMH was significantly more in maternal risk factors such as GDM, preeclampsia, placenta previa. The odd of having positive FMH was 16.45 times more in the presence of placenta previa. There was a mild negative correlation coefficient existed between the neonatal haemoglobin and amount of FMH, which was statistically significant $(\mathrm{p}$ value $=$ 0.000). That means although neonatal anaemia was not found in the babies in our study, there was a trend of having lower haemoglobin with higher FMH.

\section{CONCLUSION}

With the findings of present study, it was concluded that bigger the placenta in terms of weight and diameter more is the foetomaternal haemorrhage. Foetomaternal haemorrhage is associated with maternal complications such as multifoetal gestation, GDM, preeclampsia and a diagnostic test for FMH should be considered in such cases to detect neonatal anaemia at the earliest possible and to decide on adequate dose of anti-D to clear the foetal cells from maternal circulation.

\section{KEYWORDS}

Foetomaternal Haemorrhage (FMH), Kleihauer-Betke Test (KBT), Placental Weight and Diameter, P Values Written are Correct.

HOW TO CITE THIS ARTICLE: Reddy SV, Pai MV. Placental parameters and foetomaternal haemorrhage. J. Evolution Med. Dent. Sci. 2016;5(98):7200-7208, DOI: 10.14260/Jemds/2016/1629

Financial or Other, Competing Interest: None.

Submission 25-05-2016, Peer Review 26-11-2016,

Acceptance 02-12-2016, Published 08-12-2016.

Corresponding Author:

Dr. Muralidhar V. Pai,

Professor and HOD,

Department of Obstetrics and Gynaecology,

Kasturba Medical College,

Manipal - 576104, Karnataka.

E-mail:mvpai@manipal.edu,drmvpai@yahoo.com

DOI: $10.14260 /$ jemds/2016/1629

(c) $(7)$

\section{BACKGROUND}

Entry of foetal erythrocytes into maternal circulation before or during delivery is referred as Foetomaternal Haemorrhage (FMH). During pregnancy, normally placental barrier helps in exchange of gas and nutrients between mother and foetus. It is bilayered (Syncytiotrophoblast and cytotrophoblast). FMH occurs due to disruption in the bilayered placental barrier. The exact cause of FMH is unknown. An Rh-D negative women when exposed to the cells of an Rh-D positive foetus, produces anti-D antibodies that causes complications like foetal anaemia, hydrops foetalis and neonatal jaundice. There are a 
few diagnostic tests to detect FMH. The rosette test is qualitative, highly sensitive screening test in detecting foetal cells in maternal circulation, but it is not useful in cases with same Rh-D factor in both mother and foetus. The KleihauerBetke test is a quantitative test, which uses the concept of differential resistance to acid by foetal and adult haemoglobin. In a few cases, persistence of foetal cells is seen in the mother when there is increased transplacental cell exchange. Therefore, it is of clinical importance to identify potential risk factors for the occurrence of FMH in pregnant women to improve the neonatal outcome. As the potential risk factors can be determined by placental morphology and provide information on pregnancy outcome, they may provide information on incidence and amount of FMH also. This study was undertaken to determine the correlation between placental parameters and foetomaternal haemorrhage and also to correlate FMH with maternal factors and neonatal anaemia.

\section{MATERIALS AND METHODS}

Present study was a prospective, observational study carried out over a period of 2 years from September 2013 till September 2015 in a tertiary hospital. Prior to enrolment of patients, Ethical Committee Clearance was obtained from Institutional Ethical Committee (IEC-400/2013).

\section{Sample Size}

Assumed prevalence of FMH is taken as about $17 \% .^{3}$ Taking delivering population in the hospital as 1800 per year, sensitivity of $95 \%$, specificity of $95 \%$, desired precision of 0.05 with confidence of $95 \%$ the calculated sample size was about 317 as per the method described by Humphry RW, Cameron A and Gunn GJ, 2004.6

\section{Comment}

Any use of SPSS? Version? Yes SPSS 16.

\section{Inclusion Criteria}

Women who delivered in the hospital after 28 weeks of gestation and consented for the study.

\section{Exclusion Criteria}

Haemoglobinopathies.

All eligible women were explained the purpose of study in the language they understood and enrolled after taking written informed consent. Baseline data like maternal demographic details, blood group and type, mode of delivery, gestational age at delivery, sensitising events were noted. Maternal blood ( $2 \mathrm{~mL}$ ) was collected in EDTA bottle after the delivery of the baby. Samples were sent to Blood bank. FMH was quantified by Kleihauer-Betke's test.

\section{Kleihauer-Betke Test Method}

Principle: Foetal red cell haemoglobin $(\mathrm{Hb})$ is more resistant to acid elution than maternal red cell haemoglobin, and thus after acid treatment the maternal cells appear as ghosts due to $\mathrm{Hb}$ elution, whereas foetal cells can be stained by dyes.

\section{Materials Required}

Maternal Sample: An EDTA sample, quantity $2 \mathrm{~mL}$ with 1 in 10 dilution using normal saline within 2 hours of delivery.

\section{Baby Sample}

Cord blood sample for blood grouping.

\section{Reagents}

Methanol, Citric acid, Disodium hydrogen phosphate, Harris haematoxylin (filtered), Erythrosin B 0.5\%, Coplin Jars-3, glass slides degreased and slide spreader, Pasteur pipette and measuring cylinder.

\section{Negative Control}

Fresh EDTA blood.

\section{Positive Control}

Fresh EDTA cord blood mixed with fresh adult whole blood to a dilution of 1:100 (both should be of same blood group).

Counting was done using Mollison's formula and counted till 2000 maternal cells were counted and objective of lens was $10 \mathrm{X}$ for screening $40 \mathrm{X}$ for counting.

\section{Comment}

Was maternal sample used as whole blood or was it diluted (in NS) = 1 in 10 dilutions in normal saline.

Timing of maternal sample collection (how many hours of postpartum) $=$ within 2 hours.

How many fields were examined and objective of lens $=$ Till 2000 maternal cells were counted and Objective of lens was 10X for screening $40 \mathrm{X}$ for counting.

Name of the formula for FMH calculation = Mollison's formula.

\section{RESULTS}

The study population consisted of a total of 333 women who delivered at a tertiary hospital. Based on the results of Kleihauer-Betke's test, they were divided into two groups as follows for analysis and comparison.

Group 1 Foetomaternal Haemorrhage (FMH) positive $(>2 \mathrm{~mL})$. Group 2 FMH negative $(<2 \mathrm{~mL})$.

Out of a total of 333 subjects, $48(14.4 \%)$ were positive for FMH (Group 1) and 285 (85.6\%) were negative for FMH (Group 2).

\begin{tabular}{|c|c|c|c|}
\hline $\begin{array}{c}\text { Patient } \\
\text { Characteristics } \\
\text { (n) }\end{array}$ & $\begin{array}{c}\text { FMH } \\
\text { Positive } \\
(48) \\
\text { N (\%) }\end{array}$ & $\begin{array}{c}\text { FMH } \\
\text { Negative } \\
(285) \\
N(\%) \\
\end{array}$ & $\begin{array}{c}\mathbf{P} \\
\text { Value }\end{array}$ \\
\hline $\begin{array}{l}\text { Maternal age } \\
\text { (years) }\end{array}$ & $\begin{array}{c}28.85 \pm \\
4.45\end{array}$ & $28.21 \pm 3.74$ & 0.143 \\
\hline $\begin{array}{l}\text { Gestational age } \\
\text { at delivery } \\
\text { (weeks) }\end{array}$ & $\begin{array}{c}36.54 \pm \\
3.49\end{array}$ & $37.74 \pm 2.01$ & 0.0004 \\
\hline $\begin{array}{c}\text { Primigravida } \\
\text { (184) }\end{array}$ & $23(12.5)$ & $161(87.5)$ & \multirow[b]{2}{*}{0.269} \\
\hline $\begin{array}{c}\text { Multigravida } \\
\text { (149) }\end{array}$ & $25(16.8)$ & $124(83.2)$ & \\
\hline Singleton (325) & $45(13.8)$ & $280(86.2)$ & \multirow[b]{2}{*}{0.085} \\
\hline $\begin{array}{c}\text { Multiple } \\
\text { gestation (8) }\end{array}$ & $3(37.5)$ & $5(62.5)$ & \\
\hline \multicolumn{4}{|c|}{ Table 1. Demographic Details $(n=333)$} \\
\hline
\end{tabular}




\section{Independent ' $t$ ' Test}

Table 1 shows that there was no statistically significant difference with respect to maternal age between two groups; however, patients that were positive for FMH delivered slightly earlier $(36.54 \pm 3.49)$ and this was statistically significant $(\mathrm{p}=0.0004)$. The gestational ages at delivery ranged from 28 weeks to 40 weeks with 11 of $48 \mathrm{FMH}$ positive women being preterm. Of the 11, two had multiple gestations and 2 others had gestational diabetes, who accounted for skewing of distribution of placental weights and placental diameters in FMH positive group. There was no statistically significant correlation between FMH and either parity or the number of foetuses, even though apparently FMH positivity was more among patients with multi-foetal pregnancy (37.5\%) compared to that with singleton pregnancy $(13.8 \%)$. The number of women having multi-foetal pregnancy was very small.(8) The patients having multi-foetal pregnancy with positive FMH had bigger mean placental size $(610 \pm 45.83 \mathrm{gm})$ when compared to those having singleton pregnancy with positive FMH (502.44 $\pm 75.95 \mathrm{gm}$ ) (Table 1 ).

\begin{tabular}{|c|c|c|c|}
\hline $\begin{array}{c}\text { Placental } \\
\text { Parameters }\end{array}$ & $\begin{array}{c}\text { FMH Positive } \\
\text { (n= 48) }\end{array}$ & $\begin{array}{c}\text { FMH } \\
\text { Negative } \\
\text { (n= 285) }\end{array}$ & $\begin{array}{c}\text { P } \\
\text { value }\end{array}$ \\
\hline $\begin{array}{c}\text { Placenta } \\
\text { weight (gm) }\end{array}$ & $\begin{array}{c}508.54 \pm \\
80.34\end{array}$ & $\begin{array}{c}480.63 \pm \\
44.65\end{array}$ & 0.0005 \\
\hline $\begin{array}{c}\text { Placenta } \\
\text { diameter (cm) }\end{array}$ & $19.92 \pm 3.99$ & $18.61 \pm 2.77$ & 0.005 \\
\hline \multicolumn{3}{|c|}{ Table 2. Correlation between Placental Weight, } \\
Diameter and FMH (n=333) \\
\hline
\end{tabular}

The values were expressed in mean \pm standard deviation. Independent ' $\mathrm{t}$ ' test.

The mean placental weight $(508.54 \pm 80.34 \mathrm{gm})$ and the mean placental diameter $(19.92 \pm 3.99 \mathrm{~cm})$ were significantly more in women having positive FMH when compared to those with negative FMH. (P value $=0.0005$ for placental weight and $\mathrm{p}=0.005$ for placental diameter) (Table-2)

\begin{tabular}{|c|c|c|c|c|c|}
\hline $\begin{array}{c}\text { Placental } \\
\text { Weight }\end{array}$ & $\begin{array}{c}\text { N } \\
\text { (\%) }\end{array}$ & $\begin{array}{c}\text { FMH } \\
\text { Positive } \\
(\mathbf{4 8 )} \\
\mathbf{N}(\%)\end{array}$ & $\begin{array}{c}\text { FMH } \\
\text { Negative } \\
\mathbf{( 2 8 5 )} \\
\mathbf{N}(\%)\end{array}$ & $\begin{array}{c}\text { P } \\
\text { value }\end{array}$ & OR \\
\hline$<500 \mathrm{gm}$ & $\begin{array}{c}200 \\
(60)\end{array}$ & $14(7)$ & $\begin{array}{c}186 \\
(93)\end{array}$ & \multirow{2}{*}{0.00} & \multirow{2}{*}{4.56} \\
\hline \multirow{2}{*}{ Table 3. Correlation between Placental } \\
Weight and FMH (n=333) \\
\hline
\end{tabular}

Chi-square test

Table 3 shows that when placental weight was $\geq 500 \mathrm{gm}$, more women were positive for FMH (25.6\%) compared to when that was less than 500 gm (7\%). This finding was statistically significant ( $\mathrm{p}$ value $=0.00$ ) and the odds of having positive FMH was 4.56 times. Lightest placenta was $310 \mathrm{gm}$ and heaviest placenta was $650 \mathrm{gm}$. Correlation of FMH with different placental weight categories and different gestational age categories is explained in Table 4 and Table 5.

\begin{tabular}{|c|c|c|c|c|c|}
\hline $\begin{array}{c}\text { Placental } \\
\text { Weight (gm) }\end{array}$ & $\begin{array}{c}\text { Total (333) } \\
\mathbf{N}(\mathbf{9})\end{array}$ & $\begin{array}{c}\text { FMH Positive (48) } \\
\mathbf{N}(\mathbf{9})\end{array}$ & $\begin{array}{c}\text { FMH Negative (285) } \\
\mathbf{N}(\%)\end{array}$ & P value & OR \\
\hline $300-400$ & $15(4.5)$ & $5(10.42)$ & $10(3.51)$ & $0.033^{*}$ & 3.198 \\
\hline $400-499$ & $185(55.55)$ & $9(18.75)$ & $176(61.75)$ & $0.000^{*}$ & 0.143 \\
\hline $500-599$ & $124(37.53)$ & $28(58.33)$ & $96(33.68)$ & $0.001^{*}$ & 2.756 \\
\hline $600-699$ & $9(2.7)$ & $3(1.05)$ & $0.000^{*}$ & 13.43 \\
\hline
\end{tabular}

Chi-square test

When placental weights were categorised at increments of $100 \mathrm{gm}$ as shown in Table 4 , it was found that as the placental weights increased the incidence of positive FMH also increased significantly. When the placental weight was between 600 and 699 gm, the odds of having FMH was 13.43 times more.

\begin{tabular}{|c|c|c|c|c|c|c|}
\hline $\begin{array}{c}\text { Gestational } \\
\text { Age Group (Weeks) }\end{array}$ & $\begin{array}{c}\text { Placental } \\
\text { Weight (gm) }\end{array}$ & $\begin{array}{c}\text { FMH Positive } \\
\text { N (\%) }\end{array}$ & $\begin{array}{c}\text { FMH Negative } \\
\text { N (\%) }\end{array}$ & $\begin{array}{l}\text { Total } \\
\text { N (\%) } \\
\end{array}$ & P value & OR \\
\hline \multirow{4}{*}{ Less than 34 weeks } & $<400$ & $4(44.44)$ & $5(38.46)$ & 9 & 1.000 & 1.28 \\
\hline & $400-499$ & $2(20)$ & $7(53.85)$ & 9 & 0.203 & 0.25 \\
\hline & $500-599$ & $1(3.45)$ & $1(7.69)$ & 2 & 1 & 1.5 \\
\hline & $600-699$ & $2(22.22)$ & 0 & 2 & -- & -- \\
\hline \multicolumn{2}{|c|}{ Sub-Total } & $9(100)$ & $13(100)$ & $22(100)$ & $0.000 *$ & 4.83 \\
\hline \multirow{4}{*}{34 to 37 weeks } & $<400$ & $1(10)$ & $4(4.71)$ & 5 & 0.434 & 2.25 \\
\hline & $400-499$ & $1(10)$ & $66(77.65)$ & 67 & $0.000^{*}$ & 0.03 \\
\hline & $500-599$ & $7(70)$ & $14(16.47)$ & 21 & $0.000^{*}$ & 11.83 \\
\hline & $600-699$ & $1(10)$ & $1(1.18)$ & 2 & 0.200 & 9.33 \\
\hline \multicolumn{2}{|c|}{ Sub-Total } & $10(100)$ & $85(100)$ & $95(100)$ & 0.202 & 0.62 \\
\hline \multirow{4}{*}{ More than 37 weeks } & $<400$ & 0 & $1(0.53)$ & 1 & -- & -- \\
\hline & $400-499$ & $6(20.6)$ & $103(55.08)$ & 109 & $0.000^{*}$ & 0.21 \\
\hline & $500-599$ & $20(68.97)$ & $81(43.32)$ & 101 & $0.009^{*}$ & 2.90 \\
\hline & $600-699$ & $3(10.34)$ & $2(1.07)$ & 5 & $0.015^{*}$ & 10.67 \\
\hline \multicolumn{2}{|c|}{ Sub-Total } & $29(100)$ & $187(100)$ & $216(100)$ & 0.485 & 0.79 \\
\hline \multicolumn{2}{|c|}{ Total } & 48 & 285 & 333 & -- & -- \\
\hline
\end{tabular}


To correlate the placental weight at different gestational age with FMH, the patients were divided into categories as shown in Table 5. It was found that placentae were significantly heavier as the gestational age advanced and accordingly the incidence of positive FMH. In all gestational age groups, heavier placentae were found to have higher risk of FMH. There were more placentae weighing $>600 \mathrm{gm}$ when the gestational age was $>37$ weeks and the odds of those women having FMH was 10.67 . At $<34$ weeks 4 women had placental weight between 500 - 699 gms, they were multi-foetal gestations. At > 37 weeks 1 patient had placental weight $<400$ gms, she was found to have IUD.

\begin{tabular}{|c|c|c|c|c|c|}
\hline Placenta Diameter & N (\%) & $\begin{array}{c}\text { FMH Positive (48) } \\
\text { N (\%) }\end{array}$ & $\begin{array}{c}\text { FMH Negative (285) } \\
\text { N (\%) }\end{array}$ & $P$ value & OR \\
\hline$\geq 22 \mathrm{~cm}$ & $59(17.7)$ & $20(33.9)$ & $39(66.1)$ & \multirow{2}{*}{0.00} & \multirow{2}{*}{4.51} \\
\hline$<22 \mathrm{~cm}$ & $274(85.3)$ & $28(10.2)$ & $246(89.8)$ & & \\
\hline
\end{tabular}

Chi-square test

Table 6 shows that when the placental diameter was $\geq 22 \mathrm{~cm}$, the incidence of positive FMH was significantly more (33.9\%) compared to when that was $<22 \mathrm{~cm}(10.2 \%)$ ( $\mathrm{p}$ value $=0.00)$ and the odds of having positive FMH was 4.51 times.

\begin{tabular}{|c|c|c|c|c|c|}
\hline $\begin{array}{l}\text { Placental } \\
\text { Diameter } \\
\text { (cm) }\end{array}$ & $\begin{array}{c}\text { Total } \\
\mathrm{N}=333 \\
\mathrm{~N}(\%)\end{array}$ & $\begin{array}{c}\text { FMH } \\
\text { Positive (48) } \\
\text { N (\%) }\end{array}$ & $\begin{array}{c}\text { FMH } \\
\text { Negative (285) } \\
\text { N (\%) }\end{array}$ & P value & OR \\
\hline $13-17$ & $90(27)$ & $16(33.33)$ & $74(32.98)$ & 0.962 & 1.016 \\
\hline $18-22$ & $207(62.1)$ & $18(37.5)$ & $189(59.3)$ & 0.005 & 0.412 \\
\hline $23-27$ & $36(10.8)$ & $14(29.17)$ & $22(7.72)$ & 0.000 & 4.922 \\
\hline
\end{tabular}

Chi-square test

When placental diameters were categorised at increments of $4 \mathrm{~cm}$ as shown in Table 7, it was found that the women with placental diameter $23 \mathrm{~cm}$ and above were found to have about 5 times higher risk of FMH being positive and this was statistically significant ( $\mathrm{p}-0.00)$.

\begin{tabular}{|c|c|c|c|c|c|c|c|c|}
\hline $\begin{array}{l}\text { Gestational Age } \\
\text { Group (Weeks) }\end{array}$ & $\begin{array}{c}\text { Placental } \\
\text { Diameter } \\
\text { (cms) }\end{array}$ & $\begin{array}{c}\text { FMH } \\
+\end{array}$ & $\begin{array}{c}\text { FMH + ve as \% of } \\
\text { the Subgroup }\end{array}$ & $\begin{array}{c}\text { FMH } \\
-\end{array}$ & $\begin{array}{c}\text { FMH -Ve as \% of } \\
\text { the Subgroup }\end{array}$ & Total & OR & $\begin{array}{c}P \\
\text { value }\end{array}$ \\
\hline \multirow{3}{*}{ Less than 34 weeks } & $13-17$ & 7 & 77.78 & 11 & 84.62 & 18 & 0.636 & 0.683 \\
\hline & $18-22$ & 0 & 0.00 & 1 & 7.69 & 1 & -- & -- \\
\hline & $23-27$ & 2 & 22.22 & 1 & 7.69 & 3 & 3.429 & 0.544 \\
\hline \multicolumn{2}{|c|}{ Sub-Total } & 9 & $100 \%$ & 13 & $100 \%$ & 22 & 4.828 & 0.000* \\
\hline \multirow{3}{*}{34 to 37 weeks } & $13-17$ & 3 & 30.00 & 45 & 52.94 & 48 & 0.381 & 0.199 \\
\hline & $18-22$ & 5 & 50.00 & 36 & 42.35 & 41 & 1.361 & 0.644 \\
\hline & $23-27$ & 2 & 20.00 & 4 & 4.71 & 6 & 5.063 & 0.119 \\
\hline \multicolumn{2}{|c|}{ Sub-Total } & 10 & $100 \%$ & 85 & $100 \%$ & 95 & 0.619 & 0.202 \\
\hline \multirow{3}{*}{ More than 37 weeks } & $13-17$ & 6 & 20.69 & 18 & 9.63 & 24 & 2.449 & 0.078 \\
\hline & $18-22$ & 13 & 44.83 & 152 & 81.28 & 165 & 0.187 & $0.000^{*}$ \\
\hline & $23-27$ & 10 & 34.48 & 17 & 9.09 & 27 & 5.263 & $0.000^{*}$ \\
\hline \multicolumn{2}{|c|}{ Sub-Total } & 29 & $100 \%$ & 187 & $100 \%$ & 216 & 0.799 & 0.485 \\
\hline \multicolumn{2}{|c|}{ Total } & 48 & -- & 285 & -- & 333 & -- & -- \\
\hline \multicolumn{9}{|c|}{ Table 8. Correlation between Placental Diameter at Different Gestational Age and FMH (n = 333) } \\
\hline
\end{tabular}

Chi-square test

To correlate the placental diameter at different gestational age with FMH, the patients were divided into categories as shown in Table 8. It was found that placentae were significantly bigger as the gestational age advanced and accordingly the incidence of positive FMH was significantly more. Further, in all gestational age groups, larger placentae were found to have higher risk of FMH. There were more placentae measuring between 23 and $27 \mathrm{~cm}$ when the gestational age was $>37$ weeks and the odds of those patients having positive FMH was 5.3. In the gestational age group $<34$ weeks, 3 women with placental diameter between 23 and 27 $\mathrm{cm}$ were found to have multi-foetal gestations. In the group > 37 weeks, women with placental diameter between 13 and 17 $\mathrm{cm}$ were found to have intrauterine growth restriction in foetus and hypertension.

\begin{tabular}{|c|c|c|c|c|}
\hline Parameter & Mean & SD & $\begin{array}{c}\text { Correlation } \\
\text { Coefficient } \\
\text { (r) }\end{array}$ & $\begin{array}{c}P \\
\text { value }\end{array}$ \\
\hline $\begin{array}{l}\text { Placental } \\
\text { Weight } \\
\text { (gms) }\end{array}$ & 484.745 & 51.802 & \multirow{2}{*}{$\begin{array}{c}0.803248 \\
\text { Strong } \\
\text { Positive } \\
\text { Correlation }\end{array}$} & \multirow{2}{*}{$0.000^{*}$} \\
\hline $\begin{array}{l}\text { Placental } \\
\text { Diameter } \\
(\mathrm{cm})\end{array}$ & 18.796 & 3.010 & & \\
\hline Table 9 & $\begin{array}{r}\text { Prrelatio } \\
\text { Pla }\end{array}$ & $\begin{array}{l}\text { of Plac } \\
\text { ntal W }\end{array}$ & $\begin{array}{l}\text { al Diamet } \\
\text { ht }\end{array}$ & \\
\hline
\end{tabular}




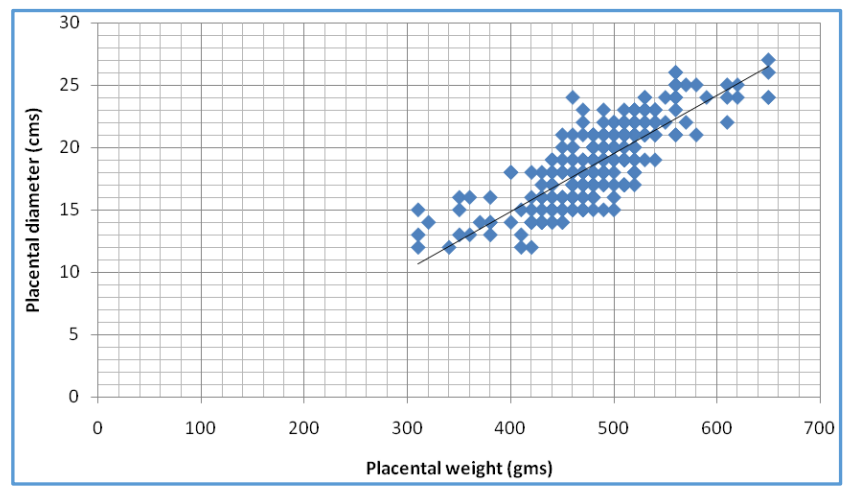

Placental diameter had strong positive correlation with Placental weight and it was statistically significant. (Table 9 and Figure 1).

Figure 1. Correlation of Placental Diameter with Placental Weight

\begin{tabular}{|c|c|c|c|}
\hline FMH & N (\%) & $\begin{array}{c}\text { Placental Weight } \\
\text { (gm) }\end{array}$ & $\begin{array}{c}\text { Placental Diameter } \\
(\mathbf{c m})\end{array}$ \\
\hline$<1 \mathrm{~mL}$ & $183(54.9)$ & $484.59 \pm 39.31$ & $18.89 \pm 2.68$ \\
\hline $1-2 \mathrm{~mL}$ & $104(31.2)$ & $473.75 \pm 52.24$ & $18.12 \pm 2.88$ \\
\hline$>2 \mathrm{~mL}$ & $48(14.4)$ & $508.54 \pm 80.34$ & $19.92 \pm 4.00$ \\
\hline \multicolumn{2}{|r|}{ Table 10. Quantification of FMH and Correlation between Placental Weight, Diameter and FMH (n = 333) } \\
\hline
\end{tabular}

Chi-square test

Table 10 shows that when the FMH was $>2 \mathrm{~mL}$, both mean placental weight $(508.54 \pm 80.34 \mathrm{gm})$ and diameter $(19.92 \pm$ $4.00 \mathrm{~cm}$ ) were significantly higher when compared to $\mathrm{FMH}<1$ $\mathrm{mL}$ and FMH between $1-2 \mathrm{~mL}$ ( $\mathrm{p}$ value 0.00 for both weight

and diameter). This again shows that as the placental weight and diameter increases, the quantity of FMH increases. The maximum FMH seen was $6.5 \mathrm{~mL}$.

\begin{tabular}{|c|c|c|c|c|}
\hline $\begin{array}{c}\text { Mode } \\
\text { of Delivery }\end{array}$ & N (\%) & $\begin{array}{c}\text { FMH Positive (48) } \\
\text { N (\%) }\end{array}$ & $\begin{array}{c}\text { FMH Negative (285) } \\
\text { N (\%) }\end{array}$ & P value \\
\hline Vaginal delivery & $100(30.03)$ & $17(17)$ & $83(83)$ & 0.379 \\
\hline LSCS & $233(69.97)$ & $31(13.3)$ & $202(60.7)$ & \\
\hline \multicolumn{2}{|c|}{ Table 11. Correlation between Vaginal or Caesarean Deliveries and FMH (n = 333) } \\
\hline
\end{tabular}

Chi-square test

\begin{tabular}{|c|c|c|c|c|c|}
\hline Mode of Delivery & $\begin{array}{c}\text { Total (333) } \\
\text { N (\%) }\end{array}$ & $\begin{array}{c}\text { FMH Positive (48) } \\
\text { N (\%) }\end{array}$ & $\begin{array}{c}\text { FMH Negative (285) } \\
\text { N (\%) }\end{array}$ & P value & OR \\
\hline Elective LSCS & $78(23.42)$ & $7(14.58)$ & $71(24.91)$ & 0.110 & 0.515 \\
\hline Emergency LSCS & $155(46.54)$ & $24(50)$ & $131(45.96)$ & 0.604 & 1.176 \\
\hline Normal Delivery & $93(27.92)$ & $15(31.25)$ & $78(27.37)$ & 0.579 & 1.206 \\
\hline Vacuum Delivery & $6(1.8)$ & $2(4.17)$ & $4(1.4)$ & 0.208 & 3.054 \\
\hline Forceps Delivery & $1(0.3)$ & 0 & $1(0.35)$ & -- & -- \\
\hline \multicolumn{2}{|r}{} \\
\hline
\end{tabular}

Chi-square test

There was no statistically significant correlation between the mode of delivery and incidence of positive FMH as shown in Tables 11 and 12.

\begin{tabular}{|c|c|c|c|c|c|}
\hline Maternal Complication & N (\%) & $\begin{array}{c}\text { FMH Positive (48) } \\
\text { N (\%) }\end{array}$ & $\begin{array}{c}\text { FMH Negative (285) } \\
\text { N (\%) }\end{array}$ & P value & OR \\
\hline Multiple Pregnancy & $8(2.4)$ & $3(37.5)$ & $5(62.5)$ & 0.093 & 3.73 \\
\hline GDM & $27(8.1)$ & $9(33.3)$ & $18(66.6)$ & $0.003^{*}$ & 3.42 \\
\hline Preeclampsia & $39(11.7)$ & $13(33.3)$ & $26(66.6)$ & $0.000^{*}$ & 3.70 \\
\hline IUD & $5(1.5)$ & $2(40)$ & $3(60)$ & 0.150 & 4.08 \\
\hline Placenta Previa & $7(2.1)$ & $5(71.4)$ & $2(28.5)$ & $0.001^{*}$ & 16.45 \\
\hline IUGR & $22(6.6)$ & $4(18.1)$ & $18(81.8)$ & 0.538 & 1.348 \\
\hline \multicolumn{2}{|c|}{ Table 13. FMH in Relation to Maternal Complications (n = 333) } \\
\hline
\end{tabular}

Chi-square test

Table 13 shows that incidence of positive FMH was significantly more in maternal risk factors such as GDM, preeclampsia and placenta previa. It was more in patients having multiple pregnancies and intrauterine death also, though not statistically significant. 
The odds of having positive FMH was 16.45 times more in the presence of placenta previa. There were 2 cases of abruption and that subject was positive for FMH.

\begin{tabular}{|c|c|c|c|c|c|}
\hline \multirow{2}{*}{ Maternal Complication } & \multicolumn{2}{|r|}{ FMH +ve } & \multicolumn{2}{|r|}{ FMH -ve } & \multirow{2}{*}{$P$ value } \\
\hline & $\mathbf{N}$ & Mean PD (cms) & $\mathbf{N}$ & Mean PD (cms) & \\
\hline Multiple Pregnancy & 3 & $25.00 \pm 1.73$ & 5 & $21.00 \pm 5.05$ & 0.122 \\
\hline GDM & 9 & $19.67 \pm 2.55$ & 18 & $19.83 \pm 2.53$ & 0.43 \\
\hline Preeclampsia & 13 & $20.38 \pm 4.81$ & 26 & $17.65 \pm 3.16$ & $0.0199^{*}$ \\
\hline IUD & 2 & $19.50 \pm 2.12$ & 3 & $14.67 \pm 2.52$ & 0.0568 \\
\hline Placenta Previa & 5 & $21.00 \pm 1.87$ & 2 & $14.00 \pm 0$ & $0.0025^{*}$ \\
\hline IUGR & 4 & $16.25 \pm 5.25$ & 18 & $15.33 \pm 2.74$ & 0.615 \\
\hline
\end{tabular}

Table 14 shows us that placental diameters were found to be more in FMH positive group compared to FMH negative group and it was found to be statistically significant in preeclampsia and placenta previa.

\begin{tabular}{|c|c|c|c|c|c|}
\hline \multirow{2}{*}{ Maternal Complication } & \multicolumn{3}{|c|}{ FMH +ve } & \multicolumn{3}{c|}{ FMH -ve } & \multirow{2}{*}{ P value } \\
\cline { 2 - 6 } & $\mathbf{N}$ & Mean PW (gms) & N & Mean PW (gms) & 0.171 \\
\hline Multiple Pregnancy & 3 & $610 \pm 45.83$ & 5 & $550 \pm 91.92$ & 0.090 \\
\hline GDM & 9 & $516.67 \pm 33.91$ & 18 & $495 \pm 40.48$ & 0.107 \\
\hline Preeclampsia & 13 & $506.92 \pm 113.31$ & 26 & $460 \pm 64.68$ & 0.151 \\
\hline IUD & 2 & $465 \pm 21.21$ & 3 & $406.67 \pm 61.1$ & $0.0029 *$ \\
\hline Placenta Previa & 5 & $512 \pm 23.87$ & 2 & $430 \pm 0$ & 0.559 \\
\hline IUGR & 4 & $392.5 \pm 119.27$ & 18 & $413.33 \pm 47.15$ & \\
\hline
\end{tabular}

Table 15 shows us that placental weights were found to be more in FMH positive group compared to FMH negative group except in IUGR cases and it was found to be statistically significant in placenta previa.

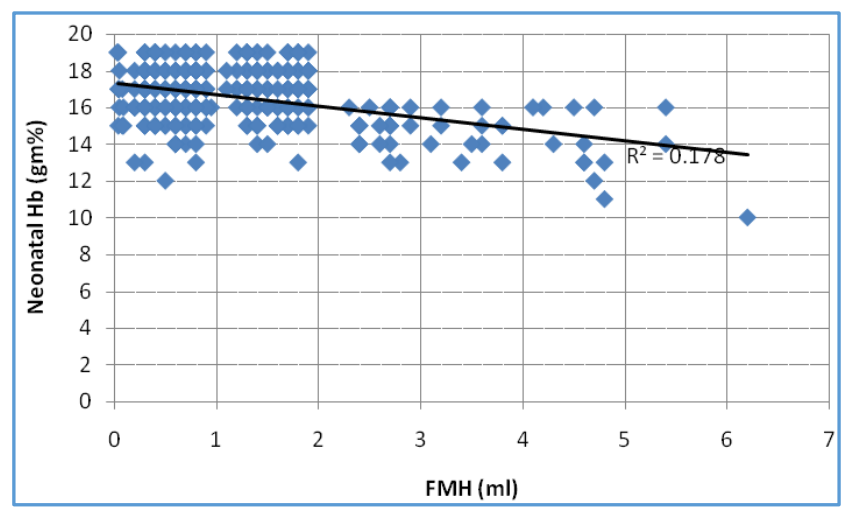

Figure 2. Correlation between FMH and Neonatal Haemoglobin $(n=333)$

\begin{tabular}{|c|c|c|c|}
\hline Parameter & Mean \pm SD & $\begin{array}{c}\text { Correlation } \\
\text { Coefficient (r) }\end{array}$ & $\begin{array}{c}\text { P } \\
\text { value }\end{array}$ \\
\hline FMH (mL) & $1.268 \pm 1.097$ & \multirow{2}{*}{$(-) 0.4228$} & $0.000^{*}$ \\
\cline { 1 - 2 } $\begin{array}{c}\text { Neonatal Hb } \\
\text { (gm } \%)\end{array}$ & $16.508 \pm 1.625$ & & \\
\cline { 1 - 2 } Table 16. Correlation between Neonatal Haemoglobin \\
and FMH (n = 333)
\end{tabular}

Figure 2 and Table 16 shows that there was a mild negative correlation coefficient existed between the neonatal haemoglobin and amount of FMH, which was statistically significant $(\mathrm{p}$ value $=0.000)$. That means although neonatal anaemia was not found in the babies in our study, there was a trend of having lower haemoglobin with higher FMH.

Incidence of FMH was found to be more in heavier and larger placenta. It was found to be more in maternal complications such as GDM, multi-foetal gestation, preeclampsia, placenta previa with antepartum haemorrhage, abruption and IUD. There was a mild negative correlation coefficient existed between the neonatal haemoglobin and amount of FMH. So in cases of heavier and larger placenta, especially in presence of maternal complications, suspect FMH and neonatal anaemia.

\section{DISCUSSION}

The entry of foetal red blood cells into maternal circulation is foetomaternal haemorrhage. Antenatal FMH is a pathological condition caused by a variety of placental and maternal factors. Secondary to the anaemia, FMH may have effects on the foetus such as hydrops foetalis, sudden unexplained term foetal death/stillbirth or neonatal death. Placental parameters provide information on pregnancy outcome including FMH.

This prospective observational study was conducted to determine the correlation between placental parameters (weight and diameter) and the incidence as well as severity of Foetomaternal Haemorrhage (FMH), and also to correlate FMH with maternal factors and neonatal anaemia.

Ideally, automated Kleihauer-Betke's Test (KBT) should be performed, as it provides accurate quantification of both small and large FMH when compared to Manual KBT. However, automated KBT was not available in our Institution, hence manual KBT was performed to determine the FMH.

Further, determining exact amount of FMH would have been better to correlate it with placental and maternal parameters as well as neonatal haemoglobin. FMH $>2 \mathrm{~mL}$ is considered as positive and less than that as negative as per BSCH guidelines. ${ }^{2}$ Hence, in the present study the patients were grouped accordingly. However, this semi-quantitative acid elution screening method has been described and validated by E Austin et al, who have opined that FMH $<2 \mathrm{~mL}$ may not always require quantification and recommended quantification to be performed if the number of foetal cells 
seen by acid elution exceeds $2 \mathrm{~mL}$ and that flow cytometry can be used to confirm these potentially significant bleeds. ${ }^{2}$

In our study, out of 333 subjects 48 were positive for FMH giving an incidence of $14.4 \%$, this was in agreement with the findings of Adetunji et al who observed FMH of $17.63 \%$ among 295 parturients of which $2.71 \%$ had large FMH (> $15 \mathrm{~mL}) .^{3}$ In our study, 285 (85.6\%) were negative for FMH. Zipursky et al quoted the incidence of FMH to be 21 to $75 \%$ of pregnancies. Renaer M et al reported $15 \%$ to $31 \%$ of pregnancies with some amount of FMH, $1.5 \%$ to $6 \%$ with volume $>0.1 \mathrm{~mL}^{8}$. Sebring and Polesky in their review of large series concluded that the volume of FMH is usually very small and the foetal red cell volume ranged from 1 to $15 \mathrm{~mL}$ in $3.7 \%$ and only $0.3 \%$ of the women had FMH larger than $15 \mathrm{~mL} .{ }^{9}$ In the present study the incidence of positive FMH ( $>2 \mathrm{~mL}$ ) was $14.4 \%$, which is higher than that observed by Renaer $\mathrm{M}$ et al and reviewed by Sebring and Polesky. This may be due to small sample size, that too consisting mostly high risk pregnancies as ours is a tertiary care hospital. High risk women such as those with preeclampsia, GDM and antepartum haemorrhage are known to have higher chance of FMH.10

There was no statistically significant difference with respect to maternal age between two groups in the present study. Age as such is not an independent factor for FMH unless it is compounded by maternal complications. Patients that were positive for FMH delivered slightly earlier $(36.54 \pm 3.49$ weeks) when compared to those that were negative for FMH (37.74 \pm 2.01 weeks). This difference was statistically significant $(\mathrm{p}$ value $=0.0004)$ suggesting $\mathrm{FMH}$ is more in women that deliver early, but this may be due to more risk factors that these women had for FMH rather than the gestational age itself. Women with high risk factors like severe preeclampsia, antepartum haemorrhage (abruption, placenta previa with heavy bleeding), IUD, multi-foetal gestation with PPROM and malpresentations and GDM with non-reassuring NST and uncontrolled high BP usually have early delivery. In the present study $13 / 48(27.08 \%)$ had preeclampsia, $9 / 48$ $(18.75 \%)$ had GDM and 5/48 (10.42\%) had antepartum haemorrhage (5/48), most of whom had early delivery. A. 0 . Adeniji, have found similar relation between these risk factors and large FMH.10 Wylie and D'Alton have opined that there is unexplained FMH in $80 \%$ of cases with volume $<30 \mathrm{~mL} .11$ They have proposed a theory of disruption of trophoblast, in which the foetal circulation is at higher pressure which pushes the foetal red blood cells into maternal circulation which explains FMH of greater than $30 \mathrm{~mL}$ in majority of unexplained cases.

There was no statistically significant correlation between FMH and either parity or the number of foetuses in our study, even though apparently FMH positivity was more among women with multi-foetal pregnancy (37.5\%) compared to that with singleton pregnancy (13.8\%). Usually, FMH is more in multi-foetal pregnancy and bigger size and area of placenta is blamed for that. In our study, the number of women having multi-foetal pregnancy was very small (8) to get a statistically significant correlation when compared to those having singleton pregnancy (325). Parity is also not an independent factor for FMH, unless it is compounded by other maternal complications.

The mean placental weight $(508.54 \pm 80.34 \mathrm{gm})$ and the mean placental diameter $(19.92 \pm 3.99 \mathrm{~cm})$ were significantly more in women having positive FMH when compared to those with negative FMH. (P value $=0.0005$ for placental weight and $p=0.005$ for placental diameter). FMH is also seen in cases of large placentae such as multi-foetal gestation, diabetes, erythroblastosis foetalis. Bigger placentae occupy larger area and have more intervillous space through which the FMH may occur. Findings of present study further reinforce the confounding interplay of maternal risk factors and placental parameters like weight and diameter as determinants of FMH. Our findings were in agreement with those of Adetunji et al. ${ }^{3}$

Further, it was also noted in the present study that when placental weight was $\geq 500 \mathrm{gm}$, more women were positive for FMH (25.6\%) compared to when that was less than $500 \mathrm{gm}$ $(7 \%)$. This finding was statistically significant $(\mathrm{p}$ value $=0.00)$ and the odds of having positive FMH was 4.56 times. Similarly, when the placental diameter was $\geq 22 \mathrm{~cm}$ the incidence of positive FMH was significantly more $(33.9 \%)$ compared to when that was $<22 \mathrm{~cm}(10.2 \%)(\mathrm{p}$ value $=0.00)$ and the odds of having positive FMH was 4.51 times. Both these findings strengthen the hypothesis that bigger the placenta more the FMH. Similar observations were made by Adetunji et al. In their study of 295 patients the $17.63 \%$ had FMH, 8/52 (2.71\%) were large FMH (> $15 \mathrm{~mL}$ ). A statistical significant correlation was seen with both the placenta weight $(\mathrm{P}<0.005)$ and diameter $(\mathrm{P}<0.042)$. In the group with placenta weight greater than 500 g, incidence was $24.12 \%$ compared to $4.17 \%$ with weight of placenta $\leq 500 \mathrm{~g}$. In the group with placental diameter $>22$, incidence of FMH was $20.81 \%$ compared to $11.23 \%$ with diameter $<22 \mathrm{~cm}^{3}$ (Tables 17 and 18). They concluded that both the placenta weight and diameter are significant predictors of FMH in patients.

\begin{tabular}{|c|c|c|c|c|}
\hline \multirow[b]{2}{*}{$\begin{array}{l}\text { Placental } \\
\text { Weight }\end{array}$} & \multicolumn{2}{|c|}{$\begin{array}{l}{\text { Adetunji et } \mathrm{al}^{3}}^{(\mathrm{n}=295)} \\
\end{array}$} & \multicolumn{2}{|c|}{$\begin{array}{c}\text { Present Study } \\
(n=333)\end{array}$} \\
\hline & $\begin{array}{c}\text {-Ve FMH } \\
(n=243) \\
N(\%)\end{array}$ & $\begin{array}{c}+ \text { Ve } \\
\text { FMH } \\
(52) \\
\text { N (\%) }\end{array}$ & $\begin{array}{c}\text {-Ve } \\
\text { FMH } \\
(285) \\
\text { N (\%) }\end{array}$ & $\begin{array}{c}\text { +Ve FMH } \\
(48) \\
N(\%)\end{array}$ \\
\hline$<500 \mathrm{gms}$ & $\begin{array}{c}92 \\
(37.8)\end{array}$ & $\begin{array}{c}4 \\
(7.6)\end{array}$ & $\begin{array}{c}186 \\
(65.2)\end{array}$ & $\begin{array}{c}14 \\
(29.16)\end{array}$ \\
\hline$\geq 500 \mathrm{gms}$ & $\begin{array}{c}151 \\
(62.1)\end{array}$ & $\begin{array}{c}48 \\
(92.3) \\
\end{array}$ & $\begin{array}{c}99 \\
(34.7) \\
\end{array}$ & $\begin{array}{c}34 \\
(70.8) \\
\end{array}$ \\
\hline P value & \multicolumn{2}{|c|}{0.002} & \multicolumn{2}{|c|}{0.00} \\
\hline & $\begin{array}{l}\text { 7. Correl } \\
\text { Weig }\end{array}$ & Ho & nl & \\
\hline
\end{tabular}

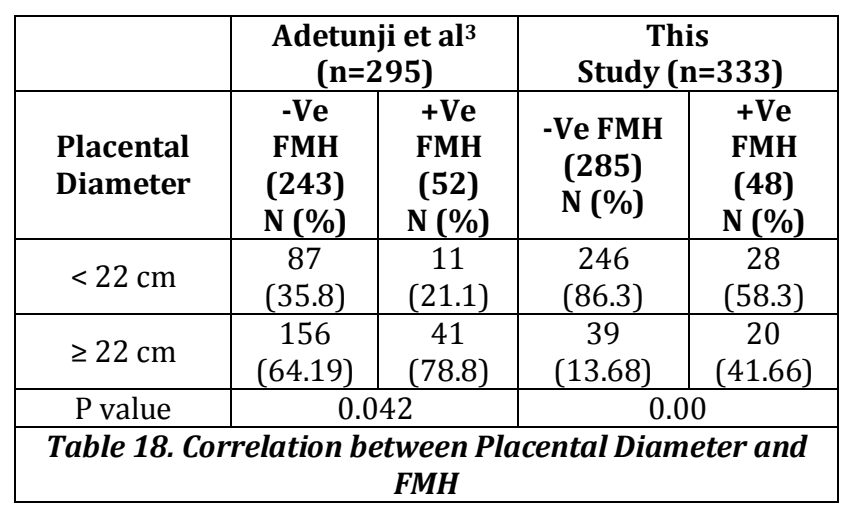

To see the strength of association between the placental weight and FMH, we correlated FMH with the placental weights at increments of 100 gm (Table 4) and found that as the placental weights increased the incidence of positive FMH also increased significantly. When the placental weight was 
between 600 and $699 \mathrm{gm}$, the odds of having FMH was 13.43 times more. When these categories of weights of placenta were correlated with FMH in 3 different gestational age groups (Table 5), we noted that in all gestational age groups heavier placentae were found to have higher risk of FMH. There were more placentae weighing $>600$ gm when the gestational age was $>37$ weeks and the odds of those women having FMH was 10.67.

Similarly to see the strength of association between the placental diameter and FMH, we correlated FMH with the placental diameter at increments of $4 \mathrm{~cm}$ (Table 7) and found that as the placental diameter increased the incidence of positive FMH also increased and when the placental diameter was between 23 and $27 \mathrm{~cm}$ the positive FMH was statistically highly significant $(p=0.000)$ and the odds of having that was 5 times more. When these categories of diameters of placenta were correlated with FMH in 3 different gestational age groups (Table 8), we noted that in all gestational age groups larger placentae were found to have higher risk of FMH. There were more placentae measuring between 23 and $27 \mathrm{~cm}$ when the gestational age was $>37$ weeks and the odds of those women having positive FMH was 5.3.

It was observed in the study that placental diameter had strong positive correlation with placental weight and it was statistically significant (Table 9 and Figure 1). Women with heavier placenta (600 - $699 \mathrm{gm})$ were found to have more odds of having positive FMH (13.43) when compared with larger placenta $(23-27 \mathrm{~cm})$, the odds ratio being 5 . It shows that heavier placenta is a more significant determinant of FMH.

Further, when the quantity of FMH was correlated with the placental parameters it was observed in our study that when the FMH was $>2 \mathrm{~mL}$ both mean placental weight (508.54 \pm $80.34 \mathrm{gm})$ and diameter $(19.92 \pm 4.00 \mathrm{~cm})$ were significantly higher when compared to FMH $<1 \mathrm{~mL}$ and FMH between $1-2$ $\mathrm{mL}$ ( $\mathrm{p}$ value 0.00 for both weight and diameter), again reinforcing the earlier observations that larger FMH is associated with larger placentae. Similar observations were made by Adetunji et al. ${ }^{3}$ The maximum FMH seen in our study was $6.5 \mathrm{~mL}$. She had antepartum haemorrhage with placenta previa type $2 \mathrm{~b}$. Baby was not affected.

All the above findings strongly suggest that bigger placentae are more often associated with FMH and the heavier the placentae the larger would be the FMH.

In the present study, there was no statistically significant correlation between the mode of delivery and incidence of positive FMH, although vacuum vaginal delivery was found to be associated with slightly higher incidence of positive FMH [2/6 (33.3\%)], but the numbers were too small to conclusively correlate. Similar findings were observed by Raed Salim, Izhar Ben-Shlomo et al. ${ }^{12}$

FMH occurs when there is trophoblastic breach and in the cases of abnormal placentation associated with preeclampsia, foetal growth restriction, GDM, intrauterine foetal death, placenta previa, placental choriocarcinomas and large placental haemangiomas (chorioangiomas). ${ }^{13,14}$ In the present study, the incidence of positive FMH was significantly more in maternal risk factors such as GDM, preeclampsia and placenta previa. It was more in women having multiple pregnancies and intrauterine death also, though not statistically significant. These were found to have heavier and larger placenta when compared to FMH negative group. The odd of having positive FMH was 16.45 times more in the presence of placenta previa.
Similar observations were made by earlier studies. ${ }^{10}$ Other case scenarios related with FMH include abruptio placentae, monochorionic-monoamniotic twin gestation. ${ }^{10,15}$ There was a case of Grade 3 abruption with retroplacental clot of 500 gms with stillborn baby and that woman was positive for FMH (4.5 $\mathrm{mL}$ ). There was another case of Grade 3 abruption with retroplacental clot of 250 gm with IUD in whom FMH was positive $(5.7 \mathrm{~mL})$. There was 1 case of MCDA twins with one twin IUD, who was positive for FMH. There was one case of Rh isoimmunisation and baby had hydrops and exchange transfusion was tried, but baby succumbed and she was positive for FMH.

Even though there were no cases of neonatal anaemia, there was a mild negative correlation coefficient existed between the neonatal haemoglobin and amount of FMH, which was statistically significant ( $p$ value $=0.000$ ). That there was a trend of having lower haemoglobin with higher FMH. The amount of FMH should be massive, more than $30 \mathrm{~mL}$ to cause neonatal anaemia in our study. The maximum FMH was 6.5 $\mathrm{mL}$. However, the negative correlation coefficient between neonatal haemoglobin and amount of FMH seen in our study shows that as the FMH increases the risk of neonatal anaemia increases as described by K. J. Moise et al. ${ }^{16}$ The choice of placental weight and diameter in this study was made, as they are mathematically related to placental surface area and volume. Along with other risk factors of FMH such as antenatal complications and procedures, placental parameters provides clue to find out the cause in unexpected mishaps.

\section{Limitations}

The study population was small. Larger sample size is needed to conclude emphatically the association between placental parameters and the amount of foetomaternal haemorrhage. Instead of defining positive or negative for FMH based on whether the haemorrhage was more than or less than $>2 \mathrm{~mL}$, assessment of exact amount of FMH would have helped to correlate the size of the placenta and quantity of FMH better. Such correlation would provide clues towards the possible cause of sudden foetal death at term due to massive FMH presumably in bigger placentae and the amount of anti-D to be given to prevent isoimmunisation and neonatal anaemia. This limitation was due to using manual Kleihauer-Betke test than automated. Though statistically significant correlation was found between the increase in quantity of FMH and declining trend of neonatal haemoglobin, the relationship between bigger placenta and occurrence of neonatal anaemia could not be established clearly as maximum FMH observed was $6.5 \mathrm{~mL}$.

\section{CONCLUSION}

With the findings of present study, it was concluded that bigger the placenta in terms of weight and diameter more is the foetomaternal haemorrhage. Foetomaternal haemorrhage is associated with maternal complications such as multi-foetal gestation, GDM, preeclampsia, placenta previa with antepartum haemorrhage, abruption and a diagnostic test for FMH should be considered in such cases to detect neonatal anaemia at the earliest possible and to decide on adequate dose of anti-D to clear the foetal cells from maternal circulation.

\section{REFERENCES}

1. Woodfield G. Guidelines for laboratory assessment of fetomaternal haemorrhage. Australian \& New zealand society of blood transfusion inc 2002. 
2. Austin E, Bates S, Howarth D, et al. Guidelines for the estimation of fetomaternal haemorrhage. BCSH FMH Guidelines, London 2009.

3. Adeniji AO, Atanda OA, Muhibi MA, et al. Role of placenta parameters in predicting significant feto-maternal haemorrhage. International Journal of Clinical Medicine 2013;4(3):133-6.

4. Sivarao S, Vidyadaran MK, Jammal AB, et al. Weight, volume and surface area of placenta of normal pregnant women and their relation to maternal and neonatal parameters in malay, Chinese and Indian ethnic groups. Placenta 2002;23(8-9):691-6.

5. Raghavendra AY, Pai V, Ramesh ST, et al. Study of placental diameter and surface area in normal and hypertensive pregnancies. Anatomica Karnataka 2013;7(3):18-22.

6. Humphry RW, Cameron A, Gunn GJ. A practical approach to calculate sample size for herd prevalence surveys. Prev Vet Med 2004;65(3-4):173-88.

7. Zipursky A, Pollock J, Neelands P, et al. The transplacental passage of foetal red blood-cells and the pathogenesis of RH immunization during pregnancy. Lancet 1963;2(7306):489-93.

8. Renaer M, de Putte VI, Vermylen C. Massive feto-maternal hemorrhage as a cause of perinatal mortality and morbidity. Eur J Obstet Gynecol Reprod Biol 1976;6(3):125-40.
9. Sebring ES, Polesky HF. Fetomaternal hemorrhage: incidence, risk factors, time of occurrence and clinical effects. Transfusion 1990;30(4):344-57.

10. Adeniji AO, Mabayoje VO, Raji AA, et al. Feto-maternal haemorrhage in parturients: incidence and its determinants. J Obstet Gynecol 2008;28(1):60-3.

11. Wylie BJ, D'Alton ME. Fetomaternal hemorrhage. Am J Obstet Gynecol 2010;115(5):1039-51.

12. Salim R, Ben-Shlomo I, Nachum Z, et al. The incidence of large fetomaternal hemorrhage and the kleihauer-betke test. J Obstet Gynecol 2005;106(3):643.

13. Aso K, Tsukimori K, Yumoto $Y$, et al. Prenatal findings in a case of massive fetomaternal hemorrhage associated with intraplacental choriocarcinoma. Fetal Diagn Ther 2009;25(1):158-62.

14. Anna-Karina AH, Maroun LL, Havsteen H, et al. Massive fetomaternal hemorrhage caused by an intraplacental choriocarcinoma: a case report. Case Rep Med 2010;2010:767218.

15. Nakamura H, Yamada Y, Akasaka J, et al. Placental abruption with certified fetomaternal hemorrhage after traffic injury. Hypertens Res Pregnancy 2014;2:33-5.

16. Moise KJ. Red blood cell alloimmunization in pregnancy. Semin Hematol 2005;42(3):169-78. 